\title{
OPTIMIZATION-BASED TUNING OF LPV FAULT DETECTION FILTERS FOR CIVIL TRANSPORT AIRCRAFT
}

\author{
D. Ossmann and A. Varga \\ German Aerospace Center (DLR) \\ Oberpfaffenhofen, Wessling 82234, Germany
}

\begin{abstract}
In this paper, a two-step optimal synthesis approach of robust fault detection (FD) filters for the model based diagnosis of sensor faults for an augmented civil aircraft is suggested. In the first step, a direct analytic synthesis of a linear parameter varying (LPV) FD filter is performed for the open-loop aircraft using an extension of the nullspace based synthesis method to LPV systems. In the second step, a multiobjective optimization problem is solved for the optimal tuning of the LPV detector parameters to ensure satisfactory FD performance for the augmented nonlinear closed-loop aircraft. Worst-case global search has been employed to assess the robustness of the fault detection system in the presence of aerodynamics uncertainties and estimation errors in the aircraft parameters. An application of the proposed method is presented for the detection of failures in the angle-of-attack sensor.
\end{abstract}

\section{INTRODUCTION}

Typical challenging problems in today's advanced flight control systems (FCS) designs for modern aircraft are aiming to significantly reduce the workload of pilots, ensuring best handling qualities simultaneously with increased passenger comfort. To increase flight autonomy, the aircraft industry traditionally uses (physical) actuator and sensor redundancy. However, this hardware-redundancy based fault detection and diagnosis (FDD) approach is becoming increasingly problematic when used in conjunction with many innovative technical solutions being developed by the aeronautical sector to satisfy the greener imperatives demanded by the society. In recent years, to alleviate this fault diagnosis bottleneck, efforts have been invested to develop FDD systems that strongly rely on advanced model-based FDD techniques. Various approaches using model-based 


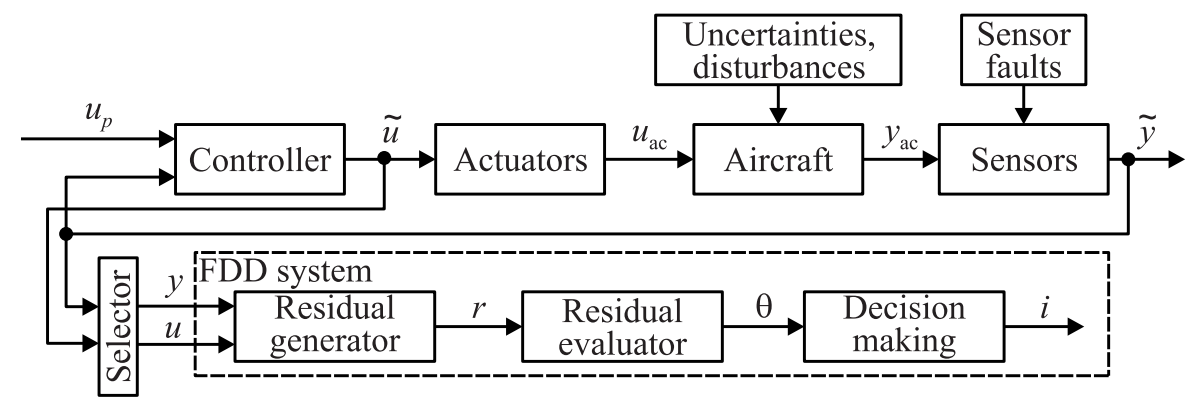

Figure 1 Augmented aircraft model architecture with FDD system

approaches are available, solving different fault monitoring tasks with increasingly higher complexity: starting from FD, which is simply a binary decision of the presence of any fault in the system, over fault detection and isolation (FDI) which localizes the fault, to fault identification (FI), which gains qualitative and quantitative knowledge about the fault.

A typical aircraft fault monitoring architecture including an FDD system is depicted in Fig. 1. The augmented closed-loop aircraft structure includes the open-loop aircraft, actuators, sensors, and a robust stabilizing controller. The FDD system structure consists of three parts [1]:

(1) a residual generator using as inputs the measurement signals $y$ and the control input signals $u$, which are derived from the measurement vector $\tilde{y}$ and controller output vector $\tilde{u}$, respectively, to generate the residual signals $r$;

(2) a residual evaluator to compute $\theta$ representing an approximation of the residual norm $\|r\|$; and

(3) a threshold-based decision making block to generate the decision signal $i$.

The main component of the FDD system is the residual generator. Generally, the fault detection problem (FDP) consists in detecting via the generated residual signals the occurrence of faults in the system. For the solution of the FDP, residual generators which are highly sensitive to all faults in the presence of controls and disturbances (also called unknown inputs) acting on the system have to be synthesized. This problem has been widely studied using different problem settings and different solution approaches. For a comprehensive account of existing methods see, for example, the monographs of [1]. Further, solving the more complex robust FDP (RFDP), when uncertainties are considered during the design of the residual generator and, therefore, an exact solution of the FDP is not possible, has been studied intensively in the last decades [2,3]. Beside these general techniques describing how to design residual generators, specific 
applications on model-based FD and residual filter design for the detection of sensor faults onboard aircraft have been developed recently. For example, the contributions $[4,5]$ provide reliable methods for the detection of sensor faults onboard an aircraft.

In this paper, a new two-step optimal synthesis approach of robust FD filters for the model-based diagnosis of sensor faults for an augmented civil aircraft is suggested. In the first step, a direct analytic synthesis of a low-order LPV FD filter is performed for the open-loop aircraft using an extension of the nullspace based synthesis method to LPV systems [6]. The main advantages of the nullspace method compared to other methods are that it allows to solve the FDP in the most general setting for both continuous- and discrete-time systems (proper or improper) and allows to determine FD filters of minimum complexity (least order). The employed scheduling parameters are either directly measurable flight conditions (altitude and speed) or aircraft parameters estimated in real time (mass and center of gravity position). In the second step, a multiobjective optimization problem is solved for the optimal tuning of the LPV detector parameters to ensure satisfactory FD performance for the augmented nonlinear closed-loop aircraft. Worst-case global search has been employed to assess the robustness of the FD system in the presence of aerodynamics uncertainties and estimation errors in the aircraft parameters. The main appeal of the proposed approach is its applicability to any robustly stable closed-loop nonlinear aircraft simulation model, for which the only requirement is that the open-loop aircraft model is trimmable and linearizable.

The FDD system must fulfill strong performance specifications which can be expressed in terms of several performance criteria. Therefore, a multiobjective optimization approach appears to be best suited for tuning the free parameters of the FDD system. Typical performance specifications are the detection time performance (DTP), missed detection rate (MDR), and false alarm rate (FAR). However, in the presence of unknown external signals (e.g., pilot inputs, wind disturbances) and parametric uncertainties, these abstract performance criteria are computationally not tractable. Therefore, the main challenge of an optimization-based design of FDD systems is to turn these performance specifications into computable optimization criteria to support the optimization-based tuning of residual generators and FDD system parameters.

An application of the suggested method is presented for the detection of failures in the angle-of-attack sensor (shortly $\alpha$-sensor) with the help of a low-order LPV-gain scheduling based detector. A first-order LPV-detector is determined by using recently proposed least order LPV-synthesis methods [6]. Due to the high complexity of the nonlinear aircraft model including highly nonlinear effects that can be encountered during certain maneuvers, in the second step, a global multiobjective parameter optimization setup is used for the optimal tuning of the residual generator parameters. The results of the LPV-detector design of the first step serve as initialization of the optimal parameter tuning. Worst-case opti- 
mizations are used in a parallel computation environment to check the robustness of the design in the defined flight range, taking also into account uncertainties in the aerodynamic database of the aircraft and in the sensor measurements.

\section{LPV-RESIDUAL GENERATOR SYNTHESIS}

In this section, the sensor RFDP is formulated for a nonlinear aircraft model and a two-step synthesis procedure of LPV residual generators is described, where the first step is a preliminary structural LPV synthesis using the openloop aircraft model, while the second step consists of an optimization-based parameter tuning employing the closed-loop robustly stable nonlinear aircraft model. The main advantage of the proposed procedure is that it is applicable to typical aircraft simulation models, where the only requirement is the trimability and linearizability of the open-loop model.

\subsection{The Robust Fault Detection Problem}

For the monitored system in Fig. 1, consisting of the open-loop aircraft, actuators, and sensors, a nonlinear state-space model description is assumed of the form

$$
\dot{\tilde{x}}(t)=F(\tilde{x}, \tilde{u}, d, \pi) ; \quad \tilde{y}(t)=G(\tilde{x}, \tilde{u}, d, \pi)+D_{f} f
$$

where $\tilde{x}$ is the state vector; $\tilde{u}$ is the control input vector; $\tilde{y}$ denotes the measured output; $d$ is an unknown disturbance vector; $f$ is the sensor fault signal; and $\pi$ is the constant parameter vector. The vector functions $F$ and $G$ are assumed to be differentiable with respect to all intervening variables, thus guaranteeing the existence of solution of (1) as well as of the various Jacobian matrices. The matrix $D_{f}$ is simply a column of the identity matrix corresponding to a selected faulty measurement.

Let $\left(\tilde{x}_{0}, \tilde{u}_{0}, \tilde{y}_{0}\right)$ be a plant equilibrium point corresponding to a fixed value of $\pi$ such that

$$
0=F\left(\tilde{x}_{0}, \tilde{u}_{0}, 0, \pi\right) ; \quad \tilde{y}_{0}=G\left(\tilde{x}_{0}, \tilde{u}_{0}, 0, \pi\right) .
$$

In a linearization-based gain scheduling approach, the first step is to obtain a linear approximation of the plant around an equilibrium point, where the (measurable) scheduling variables, denoted by $\rho_{2}$, explicitly appear. In general, $\rho_{2}$ may include the components of the measured output $\tilde{y}_{0}$ or quantities depending on $\tilde{y}_{0}$, as well as measurable parameters from $\pi$. By $\rho_{1}$, the nonmeasurable components of $\pi$ are denoted, and $\rho$ denotes the vector with stacked components $\rho_{1}$ and $\rho_{2}$. To make explicit the dependence of the equilibrium point on $\rho$, it will be denoted by $\left(\tilde{x}_{0}(\rho), \tilde{u}_{0}(\rho), \tilde{y}_{0}(\rho)\right)$. 
For the aircraft model (1), $\rho_{2}$ can be chosen with the components including the estimations of the aircraft mass $m$ and its position of center of gravity $x_{\mathrm{cg}}$, as well as the measurable outputs flight altitude $h$ and calibrated airspeed $V_{c}$. The components of $\rho_{1}$ usually include the uncertainties in the aerodynamic coefficients as well as the estimation errors in the values of $m$ and $x_{\mathrm{cg}}$.

Corresponding to the above equilibrium point, there is an LPV plant model of the form:

$$
\left.\begin{array}{l}
\dot{x}(t)=A(\rho) x(t)+B_{u}(\rho) u(t)+B_{d}(\rho) d(t) ; \\
y(t)=C(\rho) x(t)+D_{u}(\rho) u(t)+D_{d}(\rho) d(t)+D_{f} f(t)
\end{array}\right\}
$$

which describes the local behavior of the nonlinear plant around the equilibrium. In $(2), x=\tilde{x}-\tilde{x}_{0}(\rho), u=\tilde{u}-\tilde{u}_{0}(\rho)$, and $y=\tilde{y}-\tilde{y}_{0}(\rho)$, and $A(\rho)$, $B_{u}(\rho), B_{d}(\rho), D_{u}(\rho), D_{d}(\rho)$, and $D_{f}(\rho)$ are the corresponding state-, input, output- and feedthrough matrices. In what follows, it is assumed that $x(t)$ is the $n$-dimensional system state vector, $y(t)$ is the $p$-dimensional system output vector, $u(t)$ is the $m_{u}$-dimensional control input vector, $d(t)$ is the $m_{d}$-dimensional disturbance vector, and $f(t)$ is the scalar fault signal.

The vector $\rho$ is assumed to belong to a bounded region $\Pi \subset \mathcal{P}$ of the $n_{\rho^{-}}$ dimensional parameter space $\mathcal{P}$. In a more general setting, time-varying parameters $\rho(t)$ can also be allowed. However, to simplify the notations, the dependence of $\rho$ on time will not be explicitly emphasized and only slowly varying parameters which can be assimilated with constant values over sufficiently large time periods are considered.

To generate the residual signal $r(t)$ serving for the decision making on the presence or absence of a fault, a residual generator, processing the measurable output $y$ and control input $u$, has to be designed. For the envisaged synthesis, a parameter-dependent gain scheduling filter of the form

$$
\mathbf{r}(s)=Q\left(s, \rho_{2}\right)\left[\begin{array}{l}
\mathbf{y}(s) \\
\mathbf{u}(s)
\end{array}\right]
$$

is employed where $\mathbf{r}(s), \mathbf{y}(s)$, and $\mathbf{u}(s)$ are the Laplace transformed vectors $r(t)$, $y(t)$, and $u(t)$, respectively, with $r(t)$ a scalar residual signal; and $Q\left(s, \rho_{2}\right)$ is the transfer-function matrix of the filter, depending on the scheduling parameter vector $\rho_{2}$. For a physically realizable filter, $Q\left(s, \rho_{2}\right)$ must be proper with respect to the Laplace variable $s$ and robustly stable for all values of $\rho_{2}$.

The RFDP for the detection of a single sensor fault can be formulated as: Determine a proper and stable residual generator having the form (3) such that for all $\rho \in \Pi$ and given $\gamma>0$, there exists $\beta>0$ such that:

(i) $\|r(t)\| \leq \gamma \max \{\|u(t)\|,\|d(t)\|\}$ when $f=0$;

(ii) $\|r(t)\|>\beta\|f\|$ for $u=0, d=0$; and

(iii) $r(t)$ is asymptotically bounded, if $f(t)$ is asymptotically bounded. 
Here, $\|\cdot\|$ is the suitable signal norm (usually, the 2-norm). The requirement $(i)$ expresses the fact that the residual should be as small as possible in the absence of fault. If $\gamma=0$ can be used, then the residual $r$ is exactly decoupled from inputs and disturbances, and, therefore, condition $(i)$ is also called (approximate) decoupling condition. The requirement $(i i)$, called the detectability condition, ensures that the fault produces a nonzero residual. The gap defined as $\beta / \gamma$ measures the sensitivity of the detection task, where larger values guarantee the detection of smaller faults. In general, the condition (iii) on the boundedness of the residual signals requires a stable fault-to-residual transfer function matrix

$$
R_{f}(s, \rho)=Q\left(s, \rho_{2}\right)\left[G_{f}(s, \rho) 0\right]^{\mathrm{T}}
$$

where $G_{f}(s, \rho)$ is the fault-to-output transfer function matrix [7]. As the sensor fault in (2) acts directly on the output, the fault-to-residual transfer function matrix simplifies to

$$
R_{f}\left(s, \rho_{2}\right)=Q\left(s, \rho_{2}\right)\left[G_{f} 0\right]^{\mathrm{T}} .
$$

Hence, the condition (iii) is fulfilled by synthesizing a stable residual filter $Q\left(s, \rho_{2}\right)$.

\subsection{Preliminary Synthesis of an LPV-Filter}

The first step of the suggested FDD synthesis approach is the preliminary synthesis of an LPV-filter. For this purpose, $\rho_{1}$ is fixed to some representative nominal value and thus, only the case when $\rho=\rho_{2}$ is considered. For synthesis, the recently proposed multimodel-based approach of [6] is employed to determine an LPV residual filter of the form (3) which solves the RFDP. For this purpose, a family of $N$ linearized models corresponding to a discrete set of points $\Pi_{N}$ $=\left\{\rho^{(1)}, \rho^{(2)}, \ldots, \rho^{(N)}\right\}$ (obtained, for example, by parameter griding) is used to determine a family of $N$ detectors $Q\left(s, \rho^{(i)}\right)$ for $i=1, \ldots, N$. The detectors can be obtained of the form:

$$
\left.\begin{array}{c}
\dot{x}_{Q}^{i}(t)=A_{Q} x_{Q}^{i}(t)+B_{Q}\left(\rho^{(i)}\right)\left[\begin{array}{l}
y(t) \\
u(t)
\end{array}\right] ; \\
r^{i}(t)=C_{Q} x_{Q}^{i}(t)+D_{Q}\left(\rho^{(i)}\right)\left[\begin{array}{l}
y(t) \\
u(t)
\end{array}\right]
\end{array}\right\}
$$

and thus, all $N$ detectors share the same $A_{Q}$ and $C_{Q}$ matrices. This feature can be easily enforced for scalar output detectors by using a specialized version of the nullspace synthesis method of [8]. Having the grid values $B_{Q}\left(\rho^{(i)}\right)$ and $D_{Q}\left(\rho^{(i)}\right)$ for $i=1, \ldots, N$, interpolation techniques can be employed to determine parametric approximations (e.g., affine or polynomial) of matrices $B_{Q}(\rho)$ and $D_{Q}(\rho)$. The above parametric approximations can be determined along the lines of the techniques developed in [9]. 
The resulting residual generation filter has the following LPV gain scheduling form:

$$
\left.\begin{array}{c}
\dot{x}_{Q}(t)=A_{Q} x_{Q}(t)+B_{Q}(\rho)\left[\begin{array}{l}
y(t) \\
u(t)
\end{array}\right] ; \\
r(t)=C_{Q} x_{Q}(t)+D_{Q}(\rho)\left[\begin{array}{l}
y(t) \\
u(t)
\end{array}\right]
\end{array}\right\}
$$

where the matrices $A_{Q}$ and $C_{Q}$ are the constant matrices and only the matrices $B_{Q}(\rho)$ and $D_{Q}(\rho)$ depend on the parameter vector $\rho$.

The importance of the preliminary synthesis is twofold. Firstly, it serves as a good initial approximation of the final detector allowing the use of more efficient local search based optimization techniques (instead of expensive global search methods, necessary when arbitrary, e.g., random initializations, are used). Secondly, the preliminary synthesis provides a better insight into the structure of the solution of the RFDP (e.g., the zero-nonzero structures in matrices $B_{Q}(\rho)$ and $\left.D_{Q}(\rho)\right)$. This information is very helpful in reducing the number of parameters employed for detector tuning in the next synthesis step.

\subsection{Multiobjective Tuning of the LPV-Filter}

For the tuning of LPV FD filters in the second step, several performance criteria as DTP, MDR, or FAR must be simultaneously optimized. Depending on the problem to be solved, these criteria could be minimized one at a time and the rest have to remain under given bounds, or a multiobjective optimization problem can be solved. However, due to the presence of unknown external signals (e.g., pilot inputs, wind disturbances) and parametric uncertainties, these performance criteria are hardly computationally tractable, the underlying optimization problems being equivalent to optimization problems with semiinfinite constraints.

Due to the high complexity of the nonlinear aircraft model including highly nonlinear effects which can be encountered during certain maneuvers, in the second step, a multiobjective parameter optimization setup is used for the optimal tuning of the residual generator parameters. The results of the LPV-detector design of the first step serve as initialization of the optimal parameter tuning. Since the criteria evaluation involves the search over a continuous parameter space, a multimodel approach has been used which strongly favors parallel computations based expensive criteria evaluations as the simulation-based computation of FAR and MDR criteria. The DTP criterion can be included as an explicit constraint in evaluating the MDR.

To define computable approximations of the FAR and MDR criteria, simplifying assumptions are necessary. For example, the minimization of FAR can be seen to be equivalent to determine a detector which ensures the smallest $\gamma$ to 
fulfill condition $(i)$ of the RFDP. Instead of arbitrary signals $u$ and $d$, in practical applications, it is sufficient to use bounded input signals in a given finite class $\mathcal{U}$ and bounded disturbance signals in a finite class $\mathcal{D}$. A false alarm bound can be defined as

$$
J_{\text {th }}^{f}=\sup _{\substack{\rho \in \Pi \\ u \in \mathcal{U}, d \in \mathcal{D} \\ f=0}} \max _{t \leq t_{\mathrm{fin}}} \theta(t)
$$

The predefined classes $\mathcal{U}$ and $\mathcal{D}$ are typical aircraft maneuvers and disturbance signals commonly used to validate the flight controller in nonlinear simulations. The final time $t_{\text {fin }}$ is chosen in accordance with the duration of maneuvers. To avoid false alarms, the threshold $J_{\text {th }}$ used in the decision block must be chosen such that $J_{\mathrm{th}}>J_{\mathrm{th}}^{f}$.

The minimization of MDR addresses the condition ( $i i$ ) of the RFDP and can be seen equivalent to maximize the so-called detection bound defined as

$$
J_{\text {th }}^{d}=\inf _{\substack{\rho \in \Pi \\ u=0, d=0 \\ f \in \mathcal{F}}} \max _{t \leq t_{\text {det }}} \theta(t)
$$

Here, $\mathcal{F}$ is the finite set of bounded fault signals of given magnitudes which are selected to be detected within a specified detection time $t_{\text {det }}$. Note that the detection times for different fault signals in $\mathcal{F}$ may even be different. To avoid missed detections, the threshold $J_{\text {th }}$ used in the decision block must be chosen such that $J_{\text {th }}<J_{\text {th }}^{d}$. To avoid both false alarms and missed detections, the condition $J_{\text {th }}^{f}<J_{\text {th }}^{d}$ must be guaranteed via an appropriate synthesis of the FDD system.

This synthesis problem can be solved as a simultaneous minimization of $J_{\mathrm{th}}^{f}$ and maximization of $J_{\text {th }}^{d}$ over all free parameters of the FDD system. These parameters can be the free parameters of the realization (5) of the LPV detector $Q(s, \rho)$ as well as parameters $\eta$ of the evaluation block in Fig. 1 (e.g., weighting terms or filter parameters, see [10]). Thus, the following multiobjective optimization problem has to be solved $\min _{Q(s, \rho), \eta}\left[\epsilon_{1} J_{\mathrm{th}}^{f},-\epsilon_{2} J_{\mathrm{th}}^{d}\right]$ where $\epsilon_{1}$ and $\epsilon_{2}$ are the suitable scaling weights.

The evaluation of bounds (6) and (7) involves the solution of global optimization problems with respect to the parameter $\rho \in \Pi$ and, therefore, is computationally intensive. To speed up the function evaluations, it is often possible when solving synthesis problems to replace the continuous search domain $\Pi$ by a finite domain $\Pi_{N}=\left\{\rho^{(1)}, \ldots, \rho^{(N)}\right\}$ containing $N$ representative points. This replacement also facilitates the use of parallel computations when evaluating the above bounds using simulation-based runs. 


\subsection{LPV-Filter Robustness Assessment and Threshold Determination}

In the above formulation of the synthesis of the FD filter, the robustness aspects with respect to the parametric uncertainties in $\rho_{1}$ (e.g., in the aerodynamic coefficients and in the estimated values of scheduling variables) have not been explicitly addressed. Therefore, in general, for an extended parameter space $\mathcal{P}$ including all uncertainties (i.e., $\rho_{1}$ and $\rho_{2}$ ), the corresponding bound $J_{\text {th }}^{f}$ is higher, while $J_{\text {th }}^{d}$ is smaller. The decision threshold can have any value $J_{\text {th }}$ satisfying

$$
J_{\text {th }}^{f} \leq J_{\text {th }}<J_{\text {th }}^{d} .
$$

Such a choice is always possible by increasing the size of minimum amplitude of faults to be detected. A choice of threshold as $J_{\mathrm{th}}=J_{\mathrm{th}}^{f}+\varepsilon$, with a small positive $\varepsilon$, allows the detection of smaller faults.

For the computation of $J_{\mathrm{th}}^{f}$ and $J_{\mathrm{th}}^{d}$, global worst-case optimization problems have to be solved to find the worst-case parameter combinations. As a robustness measure of the FD performance, the smallest detection gap $J_{\mathrm{th}}^{d}-J_{\mathrm{th}}^{f}$ can be used, or alternatively the largest value of the detection factor

$$
\nu:=\frac{J_{\mathrm{th}}^{f}}{J_{\mathrm{th}}^{d}}
$$

If $J_{\mathrm{th}}^{d}-J_{\mathrm{th}}^{f}>0$ (or, equivalently, $\nu<1$ ), a constant threshold can be chosen to serve for FD, ensuring no false alarms and no missed detections.

Occasionally, a weaker robustness condition can be used for the determination of the decision threshold $J_{\mathrm{th}}$. A modified detection factor can be defined as

$$
\bar{\nu}:=\sup _{\rho \in \Pi} \mu(\rho) \leq \nu
$$

where $\mu(\rho)$ is the parameter-dependent detection factor defined as

$$
\mu(\rho)=\frac{\mu_{f}(\rho)}{\mu_{d}(\rho)}
$$

with

$$
\mu_{f}(\rho)=\sup _{\substack{u \in \mathcal{U}, d \in \mathcal{D}, d \\ f=0}} \max _{t \leq t_{\mathrm{fin}}} \theta(t) ; \quad \mu_{d}(\rho)=\inf _{\substack{u=0, d=0 \\ f \in \mathcal{F}}} \max _{t \leq t_{\mathrm{det}}} \theta(t) .
$$

If $\nu>1$, but $\bar{\nu}<1$, then a tolerance $J_{\mathrm{th}}\left(\rho_{2}\right)$ depending on the scheduling parameters $\rho_{2}$ can be used to solve the RFDP. 


\subsection{Discussion of the Suggested Approach}

The suggested two-step approach for the synthesis of LPV FD filters addresses the FD problem with minimal modeling requirements. To generate a family of linear models for detector synthesis at Step 1, the only computations needed are the repeated trimming and linearization of the open-loop aircraft model. These features are usually available for any nonlinear aircraft simulation model. For the detector synthesis, ready to use tools are available in the FAULT DetECTION Toolbox [7], which can be used to generate a family of detectors to serve for building LPV FD filters. If the underlying domains for the flight conditions and parameter variations are not too large, simpler LPV filters (e.g., with affine dependence of scheduling variables) can be employed. Since the matrices $A_{Q}$ and $C_{Q}$ of the detector (5) are constant, they can be fixed for the synthesis and any other tuning is performed only on the parameters of the remaining matrices of the LPV-model (5). Note that $A_{Q}$ and $C_{Q}$ can be easily determined from a single nominal design (using, for example, the least order nullspace-based synthesis method of [8]), and the resulting detector can be used for the initialization of the tuning process at Step 2. Thus, performing Step 1 can be even skipped. However, this may have also some drawbacks, since useful structural information (e.g., zero-nonzero patterns in the LPV-synthesis) are possibly lost, thus more involved optimizations problems with larger number of free parameters can result.

The multiobjective optimization-based synthesis at Step 2 can be performed using standard tools like the MOPS (multiobjective parameter search) environment of DLR [11]. The multicase feature of MOPS naturally converts any multiobjective optimization problem with $m$ criteria into a multi-objective optimization with $m N$ objective functions, where $N$ is the number of parameter points in $\Pi_{N}$ chosen for the synthesis. Since all function evaluations can be performed in parallel, parallel computations can be employed to alleviate the associated computational burden to expensive simulation runs.

\section{DETECTION OF $\alpha$-SENSOR FAULTS}

In this section, the application of the suggested approach to a nonlinear model of a closed-loop aircraft including a nonlinear control law ensuring robust stability over the whole flight envelope is described. This model was previously used in a flight control law clearance study and is described in [12]. As scheduling variables, the altitude $h$, the calibrated speed $V_{c}$, the mass $m$, and the position of the center of gravity $x_{\mathrm{cg}}$ are selected, thus, $\rho_{2}=\left(h, V_{c}, m, x_{\mathrm{cg}}\right)$. The uncertain parameters included in the vector $\rho_{1}$ are the aerodynamic coefficients $C_{x}, C_{y}$, $C_{z}, C_{m}, C_{n}$, and $C_{l}$, and the estimation errors $\Delta m$ and $\Delta x_{\mathrm{cg}}$ in $m$ and $x_{\mathrm{cg}}$, respectively. Thus, $\rho_{1}$ has 8 components whose nominal values are zero. For the 
Table 1 Values of the envelope grid

\begin{tabular}{lccc}
\hline \multicolumn{1}{c}{ Parameter } & Maximum & Minimum & Stepsize \\
\hline Altitude, ft & 15000 & 25000 & 5000 \\
Calibrated airspeed, kts & 260 & 320 & 60 \\
Mass, t & 180 & 220 & 20 \\
Center of gravity, \% & 27 & 33 & 6 \\
\hline
\end{tabular}

Table 2 Set $\mathcal{F}$ of sensor faults to be detected

\begin{tabular}{ll}
\hline \multicolumn{1}{c}{ Failure type } & \multicolumn{1}{c}{ Parameter } \\
\hline Oscillatory failure & With an amplitude of $5^{\circ}$ at $0.5 \mathrm{~Hz}$ \\
Sensor freezing & At $10^{\circ}$ \\
Slow sensor drift & With slope of $4 \mathrm{deg} / \mathrm{s}$ \\
Fast sensor drift & With slope of $15 \mathrm{deg} / \mathrm{s}$ \\
Excessive sensor noise & Of variance $\sigma^{2}=80^{\circ}$ \\
\hline
\end{tabular}

purpose of illustration of the approach, a restricted flight envelope for $\left(h, V_{c}\right)$ and a restricted domain of variation for $\left(m, x_{\mathrm{cg}}\right)$ are selected. A grid with $N=36$ grid points is used to generate nominal linearized models. The limits of the chosen envelope and the stepsize between these limits, defining the 36 grid points, are given in Table 1. The detection of sensor faults is an important issue for aircraft certification. Typical sensors faults to be detected are low-frequency oscillations around the measured values, sensor freezing [13] with the sensor providing a constant value, excessive noise (or loss of signal), drift, etc. The exact fault specifications used in this study are given in Table 2. For these faults, a maximum detection time of $t_{\mathrm{det}}=1 \mathrm{~s}$ is required. In the absence of faults, the robust FD filter must work without false alarms in all flight situations. For the estimation of the false alarm bound, a finite set of maneuvers $\mathcal{U}$ has been selected. Typical maneuvers used are, for example, piloted flights with various pilot inputs (longitudinal/lateral stick doublets, pedal input demand, noseup/nosedown demands) or typical navigation maneuvers (level flight, flight path angle target mode, yaw angle target mode, speed change, steady sideslip, coordinated turn, etc.). Typical disturbance inputs used to define $\mathcal{D}$ are random wind speeds with given variances.

From a fault tolerant control perspective, it is important to detect erroneous behavior of the sensor such that no unintended values are used in the feedback control laws. For a main sensor, as the $\alpha$-sensor, triple hardware redundancy is usually provided, which allows to easily detect single sensor faults using a voting based approach. However, the occurrence of two simultaneous $\alpha$-sensor 
faults, although highly improbable, may lead to decreased performance and may require reconfiguration of the control laws. The detection of two simultaneous faults is more challenging. The present authors' approach to this is to monitor independently each of the three sensors using a model-based FD approach and detect the $\alpha$-sensor faults using some of the existing measurements and control signals. In this way, all sensor failures can be independently detected and only the fault-free measurements are used in the control laws. The extreme case, when all three sensors fail, can be easily detected and control reconfiguration can be immediately triggered. Alternatively, an estimation of the $\alpha$-sensor measurement can be used to replace the true measurements (this possibility is not further explored in this paper).

\subsection{Preliminary LPV-Filter Synthesis}

The nonlinear open-loop aircraft model has 10 state variables, 22 control inputs, 3 disturbance inputs, and 30 measurements. For the synthesis of scalar fault detectors, a restricted set of inputs and outputs was used: 5 control inputs which have direct influence on the angle of attack $\alpha, 3$ disturbance inputs, and 6 measured outputs. The rest of variables are assumed to be constant at their trimming values. The inputs vectors $y$ and $u$ of the detector are defined as

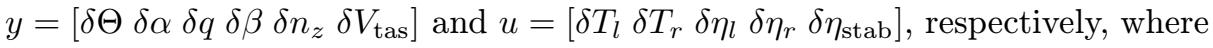
the meanings of individual variables are described in Table 3 . The generated 36 linearized models have been employed to produce 36 first-order scalar output detectors of the form (4), which served for the generation of a LPV-detector of the form (5) with constant matrices $A_{Q}=-10$ and $C_{Q}=1$, and the rest of matrices in (5) depending affinely on the scheduling variables $\rho_{2}=\left(h, V_{c}, m, x_{\mathrm{cg}}\right)$. Recall that in the preliminary synthesis, the nonmeasurable uncertainties were fixed to their nominal values $\rho_{1}=0$.

Table 3 Residual generator input signals

\begin{tabular}{ll}
\hline Input signals & \multicolumn{1}{c}{ Description } \\
\hline$\delta \Theta$ & Pitch angle \\
$\delta \alpha$ & Angle of attack \\
$\delta q$ & Pitch rate \\
$\delta \beta$ & Sideslip angle \\
$\delta n_{z}$ & Vertical load factor \\
$\delta V_{\text {tas }}$ & True airspeed \\
$\delta T_{l}, \delta T_{r}$ & Commanded left and right thrust \\
$\delta \eta_{l}, \delta \eta_{r}$ & Commanded left and right elevator deflection \\
$\delta \eta_{\text {stab }}$ & Commanded stabilizer deflection \\
\hline
\end{tabular}




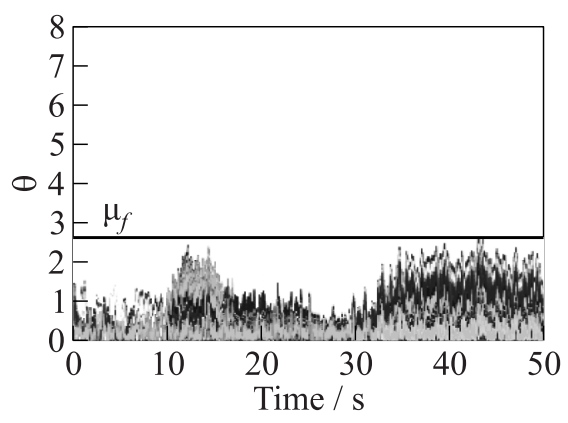

(a)

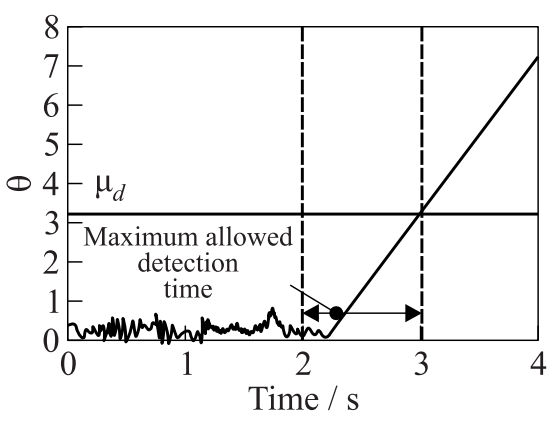

(b)

Figure 2 Detectability factor $\mu<1$ at grid point \#3

Figure 2 shows the determination of the detectability factor (10) by simulations for the grid point $\# 3$, corresponding to $m=180 \mathrm{t}, x_{\mathrm{cg}}=27 \%, \mathrm{~h}$ $=20,000 \mathrm{ft}$, and $V_{c}=260 \mathrm{kts}$. In Fig. $2 a$, fault-free cases are depicted, where the aircraft is flown through the sets of maneuvers $\mathcal{U}$ and disturbances $\mathcal{D}$. The evaluation signal $\theta(t)$, which approximates the 2-norm of the residual signal, remains rather low for all maneuvers and disturbance inputs. In Fig. $2 b, \theta(t)$ resulting from the slow $\alpha$-sensor drift (see Table 2 ) is depicted. As soon as the sensor signal starts drifting away at $t=2 \mathrm{~s}$ in the simulation, $\theta(t)$ increases dramatically, allowing the detections of the fault in the required time. The slow drift of the sensor signal is chosen as an example, as it is usually the most difficult fault to detect due to slow deviation rate. The high amplitudes and high fault rates of the remaining sensor faults described in Table 2 would lead to the stronger robustness condition $\nu<1$, enabling an adequate selection of the threshold $J_{\text {th }}$ and ensuring no false alarms and missed detections. However, for the slow sensor drift, the points in the defined range of the envelope can be found where the detectability factor $\mu>1$, avoiding the suitable selection of $J_{\text {th }}$. Although the example in Fig. 2 shows satisfactory behavior in some of the 36 design points, $\mu>1$ has been detected. To check how severe this phenomenon is within the flight range, a worst-case optimization was performed to find the bad values of $\mu>1$ and the worst-case $\bar{\nu}$. The worst-case search optimization was performed with the global search method differential evolution [11].

The results of the worst-case search are presented in Fig. 3. Several regions in the selected flight range are encountered where the detectability factor $\mu$ exhibits values greater than 1 . The criterion values are depicted with different colors, starting from good values $(\mu \leq 0.5)$ in blue, passing through cyan $(0.5$ $<\mu \leq 0.75)$, yellow $(0.75<\mu \leq 1)$ to bad values in red $(\mu>1)$. The worstcase of the detectability factor was $\bar{\nu}=1.327$ found at $m=220 \mathrm{t}, x_{\mathrm{cg}}=33 \%$, $h=25,000 \mathrm{ft}, V_{c}=317 \mathrm{kts}$, and $\mathrm{Ma}=0.73$. It follows that the FDD system 

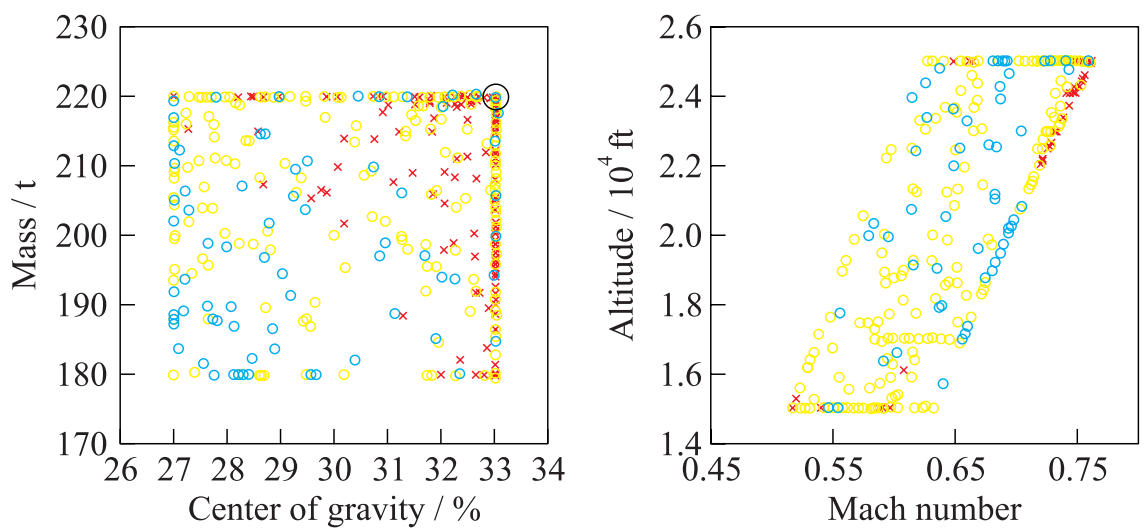

Figure 3 Worst-case search results: points in red violate the robustness requirement $\mu<1$.

based on the preliminary LPV detector does not fulfill the requirements regarding FAR, MDR, and DTP.

To increase the performance of the residual generator in respect of the FDD system and comply with the requirements of FAR, MDR, and DTP for the whole set $\mathcal{F}$ of sensor faults defined in Table 2, a setup for the optimal tuning of the detector parameters is described in the following subsection. An LPV detector with the same structure as the filter presented in this subsection is optimized using the parameter values of the nullspace synthesis as initialization for the tuning.

\subsection{Optimal Tuning of the LPV Detector}

In this section, the approach used for the optimal tuning of the LPV residual filter parameters is described. The proposed tuning approach is restricted to the region in Table 1; however, it can be easily used to cover the full flight envelope by employing several robust residual generators over similar restricted regions. For the optimal tuning of the parameters of the LPV FD filter, derivative-free local search optimization software tools were used. This was possible taking into account the preliminary synthesis results described previously. The employed optimization tools are available in the MOPS environment of DLR.

The structure of the LPV residual generator as well as the matrices $A_{Q}=-10$ and $C_{Q}=1$ are kept as designed during the linear synthesis. The free parameters of the optimization are the polynomial coefficients of the parameter-dependent matrices $B(\rho)$ and $D(\rho)$. For the 11 detector inputs, this amounts to 110 parameters which can be subject to further tuning. To alleviate the computational 
burden associated with large dimensional search spaces, there was exploited the problem structure to reduce the number of parameters subject to tuning. Firstly, all zero elements in the matrices $B(\rho)$ and $D(\rho)$ were frozen to zero values and thus excluded from optimization. Secondly, only the coefficients multiplying the outputs for which the residual is most sensitive in faulty and nonfaulty cases were selected. These are two measurements $\delta \alpha$ and $\delta q$ which mainly describe the short period dynamics of the aircraft's longitudinal motion, and $\delta \beta$ which plays an important role during maneuvers including greater rudder deflections. Additionally, from aircraft symmetry considerations, the inputs $\delta \eta_{l}$ and $\delta \eta_{r}$ share the same coefficients. In this way, the number of free parameters has been reduced to 21 , which allowed using the efficient derivative-free optimization methods as the pattern search.

The optimization is a multicase problem [11], as the continuous search domain $\Pi$ in (6) and, respectively, (7) is replaced by a finite domain $\Pi_{N}$ $=\left\{\rho^{(1)}, \ldots, \rho^{(N)}\right\}$ containing the $N=36$ representative grid points given in Table 1. This replacement also facilitates the use of parallel computations. However, during the a posteriori robustness analysis, the continuous search domain $\Pi$ is considered by using worst-case optimization techniques. An inclusion of such techniques during the optimization would directly guarantee the robustness of the synthesis by fulfilling all criteria, yet would lead to a tremendous increase of the computational burden. The optimization runs are performed applying the options for the local pattern search method [11]. The optimization run itself was performed on a Linux-cluster using 16 CPUs. A typical optimization run involving about 1300 function evaluations lasted about $54 \mathrm{~h}$.

For the assessment of the performance of the resulting FDD system, the detectability factor (9) was determined within the defined flight region. To find the flight envelope parameter values corresponding to $\bar{\nu}$, a worst-case optimization as described in subsection 3.1 was performed. The diagrams in Fig. 4 show the values of the detectability factor $\mu$ in the flight envelope and the mass and balance diagram, respectively, where the worst-case of $\bar{\nu}=0.74$ found at $m=220 \mathrm{t}$, $x_{\mathrm{cg}}=33 \%, h=15000 \mathrm{ft}, V_{c}=283 \mathrm{kts}$, and $\mathrm{Ma}=0.56$ is encircled. Thus, a reliable detection of the fault is possible without false alarms and missed detections. To clarify if a constant threshold can be used, the detectability factor (8) was determined for the optimized residual generator. The result of the worst-case search gives a value of $\nu=0.75$, allowing the selection of a constant threshold in the decision-making process. Note that in this case, $\nu \approx \bar{\nu}$. This is due to the assumption that the fault appears during cruise, where the residual signal is not influenced by any inputs $u$ and remains low regardless in which flight condition the aircraft is flown. This leads to a similar initial residual signal during the occurrence of a fault.

Finally, uncertainties in the aerodynamic coefficients in the aircraft model up to $\pm 5 \%$ are taken into account, as well as uncertainties of up to $\pm 10 \%$ in the estimations of the mass and position of the center of gravity. Figure 5 illustrates 

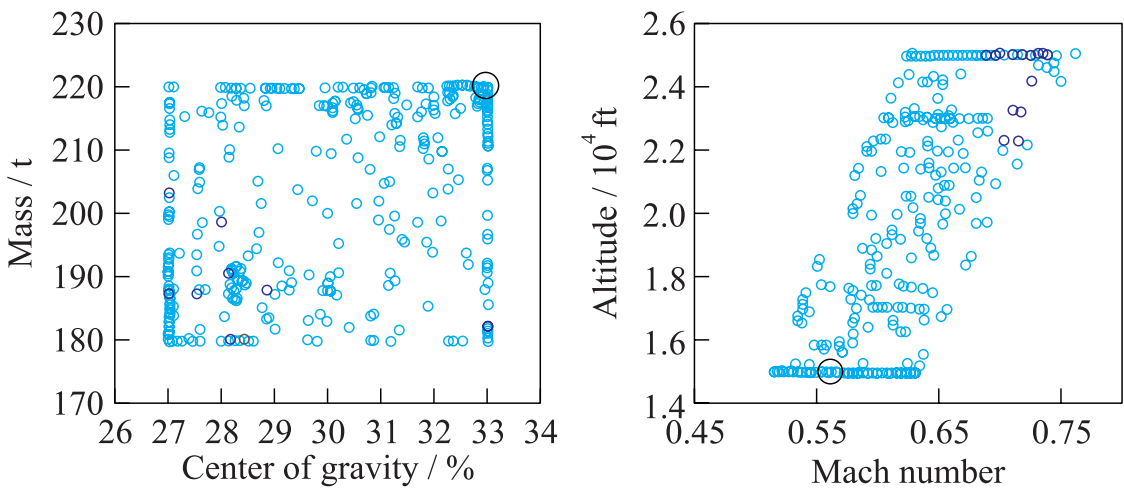

Figure 4 Worst-case search results for the nominal value $\rho_{1}=0$.
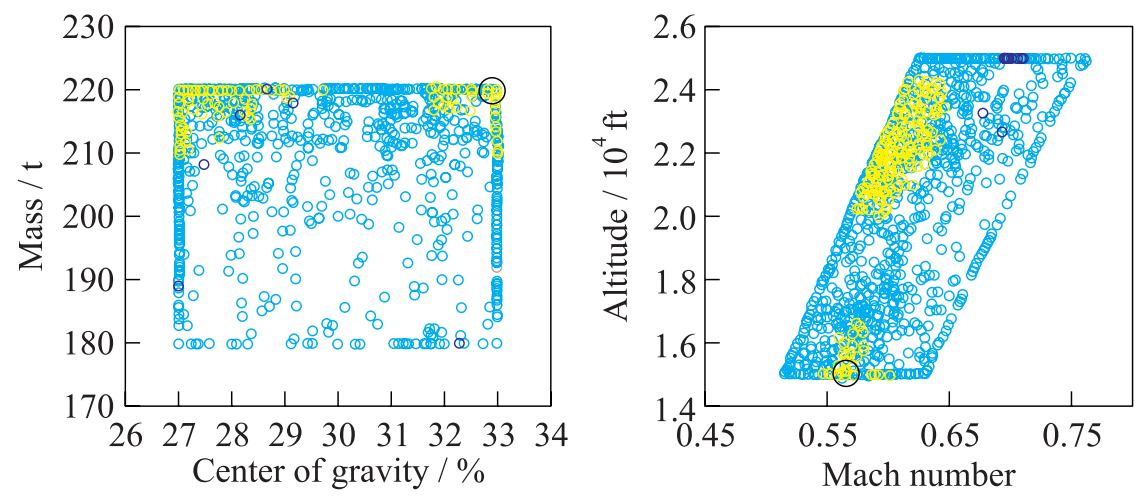

Figure 5 Worst-case search results for $\bar{\nu}$ including aerodynamic uncertainties and estimation errors.

the worst-case search result of $\bar{\nu}=0.79$ for the detectability factor found at $m=220 \mathrm{t}, x_{\mathrm{cg}}=33 \%, h=15,000 \mathrm{ft}, V_{c}=282 \mathrm{kts}$, and $\mathrm{Ma}=0.56$. As expected, due to the presence of uncertainties, the value of $\bar{\nu}$ is higher than that for the nominal case. However, no values greater than 1 were found in the whole envelope for the detectability factor $\mu$. Hence, the detection of the sensor fault is possible within the required detection time without triggering false alarms.

The worst-case search for the robustness measure (8) results in a value of $\nu$ $=0.80$, indicating a robust FD system, for which a constant threshold is selectable. For example, the selection of the false alarm bound as threshold 
Table 4 Worst-cases of the detections times for different faults

\begin{tabular}{lc}
\hline \multicolumn{1}{c}{ Failure type } & Detection time, $\mathrm{s}$ \\
\hline Oscillatory failure & 0.28 \\
Sensor jamming & 0.04 \\
Slow sensor drift & 0.88 \\
Fast sensor drift & 0.29 \\
Excessive sensor noise & 0.02 \\
\hline
\end{tabular}

$J_{\mathrm{th}}=J_{\mathrm{th}}^{f}$ leads to the worst-case detection times for the individual faults presented in Table 2 listed in Table 4. As mentioned before, the slow sensor drift is the fault which is the most difficult to detect. This is also expressed by the worst-case detections times of the different faults, as the slow sensor drift has the highest detection time. Nevertheless, after the optimization, all defined sensor faults can be detected within the required detection time without triggering false alarms or missed detections, and thus, a robust and reliable FDD system can be synthesized for the $\alpha$-sensor FD problem.

The worst-case detection times are valid for the fixed set $\mathcal{F}$ of faults given in Table 2. Decreasing fault amplitudes and/or slopes may increase the resulting detection times. However, the presented set of faults can be seen as definition of the minimum fault amplitudes/slopes for which the detection can be guaranteed. Note that the times given in Table 4 refer to pure detection times, as it is assumed that as soon as a fault is detected, the associated sensor is switched off and other (valid) signals are used instead. As in this case no discrimination of the fault types is necessary, fault identification is not considered.

\section{CONCLUDING REMARKS}

The main advantage of the suggested optimal tuning approach of the free parameters of an LPV residual generator for sensor faults is the possibility to perform the tuning using only the provided closed-loop simulation model augmented with the FDD system components. Moreover, for tuning purposes, widely available standard software tools (for trimming, simulation, and optimization) are only required. In this way, the use of analytic robust synthesis techniques relying on expensive LPV-modeling of aircraft dynamics can be completely circumvented. This is especially appealing taking into consideration the data confidentiality restrictions of aircraft manufacturers (e. g., regarding controller structure, protection laws, sensor block). This approach could thus provide a way to face the main challenge of any FDD synthesis: the availability of suitable synthesis models. 


\section{REFERENCES}

1. Gertler, J. 1998. Fault detection and diagnosis in engineering systems. New York: Marcel Dekker.

2. Patton, R. J. 1994. Robust model-based fault diagnosis: The state of the art. IFAC Symposium SAFEPROCESS'94 Proceedings. Espoo, Finland. Vol. 1.

3. Chen, J., and R. J. Patton. 1999. Robust model-based fault diagnosis for dynamic systems. London: Kluwer Academic Publs.

4. Alwi, H., C. Edwards, and A. Marcos. 2012. Fault reconstruction using a LPV sliding mode observer for a class of LPV systems. J. Franklin Inst. 349(2):510-30.

5. Berdjag, D., J. Cieslak, and A. Zolghadri. 2013. Fault detection and isolation of aircraft air data/inertial system. In: Progress in flight dynamics, guidance, navigation, control, fault detection, and avionics. Eds. Ch. Vallet, D. Choukroun, Ch. Philippe, G. Balas, A. Nebylov, and O. Yanova. EUCASS advances in aerospace sciences book ser. Moscow: TORUS PRESS. 6:317-32.

6. Varga, A. 2011. Synthesis of robust gain scheduling based fault detection filters for a class of parameter uncertain nonlinear systems. MED'11 Proceedings. Corfu, Greece.

7. Varga, A. 2008. Linear FDI-techniques and software tools. Fault Detection Toolbox V0.8 - Technical Documentation IB 515-08-18. German Aerospace Center (DLR), Institute of Robotics and Mechatronics.

8. Varga, A. 2007. On designing least order residual generators for fault detection and isolation. 16th Conference (International) on Control Systems and Computer Science Proceedings. Bucharest, Romania. 323-30.

9. Pfifer, H., and S. Hecker. 2008. Generation of optimal linear parametric models for LFT-based robust stability analysis and control design. IEEE CDC'09 Proceedings. Cancun, Mexico.

10. Ossmann, D., S. Hecker, and A. Varga. 2010. A versatile simulation environment of FTC architectures for large transport aircraft. ICAS'10 Proceedings. Nice, France.

11. Joos, H.-D., J. Bals, G. Looye, K. Schnepper, and A. Varga. 2002. A multi-objective optimisation-based software environment for control systems design. CACSD'2002 Proceedings. Glasgow, U.K.

12. Puyou, G., and Y. Losser. 2011. Clearance benchmark for a civil aircraft. In: Optimization based clearance of flight control laws. Eds. A. Varga, A. Hanson, and G. Puyou. Lecture notes in control and information sciences ser. Berlin: SpringerVerlag.

13. Boskovic, J.D., and R. K. Mehra. 2003. Fault diagnosis and fault tolerance for mechatronic systems: Recent advances. Springer-Verlag. 